\title{
Effective spin-orbit interaction Hamiltonian for quasi-one-dimensional quantum rings
}

\author{
Kh. Shakouri, ${ }^{1}$ B. Szafran, ${ }^{2}$ M. Esmaeilzadeh, ${ }^{1}$ and F. M. Peeters ${ }^{3}$ \\ ${ }^{1}$ Department of Physics, Iran University of Science and Technology, Narmak, Tehran 16844, Iran \\ ${ }^{2}$ AGH University of Science and Technology, Faculty of Physics and Applied Computer Science, al. Mickiewicza 30, 30-059 Kraków, Poland \\ ${ }^{3}$ Departement Fysica, Universiteit Antwerpen, Groenenborgerlaan 171, B-2020 Antwerpen, Belgium
}

(Received 5 January 2012; revised manuscript received 17 March 2012; published 20 April 2012; corrected 26 April 2012)

\begin{abstract}
The effective Hamiltonian for an electron in a quasi-one-dimensional quantum ring in the presence of spin-orbit interactions is derived. We demonstrate that, when both coupling types are simultaneously present, the effective Hamiltonian derived by the lowest-radial-state approximation produces energy spectra and charge densities which deviate strongly from the exact ones. For equal Rashba and Dresselhaus coupling constants the lowest-radial-state approximation opens artifactal avoided crossings in the energy spectra and deforms the circular symmetry of the confined charge densities. In this case, there does not exist a ring thin enough to justify the restriction to the lowest radially quantized energy state. We derive the effective Hamiltonian accounting for both the lowest and the first excited radial states, and show that the inclusion of the latter restores the correct features of the exact solution. Relation of this result to the states of a quantum wire is also discussed.
\end{abstract}

DOI: 10.1103/PhysRevB.85.165314

PACS number(s): 73.63.Nm

\section{INTRODUCTION}

In semiconductor nanostructures containing a twodimensional electron gas spin-orbit (SO) interactions appear due to the asymmetry of the structure ( Rashba $^{1}$ SO coupling) or of the host crystal (Dresselhaus ${ }^{2}$ coupling). SO coupling is extensively studied for its role in the relaxation, dephasing, and spin manipulation in orbital degrees of freedom. ${ }^{3,4}$ Special attention has been addressed to quantum rings ${ }^{5-21}$ in which the SO coupling - the gate voltage controlled Rashba interaction ${ }^{22}$ in particular-introduces spin interference phenomena ${ }^{5}$ that are considered for quantum information processing applications. The theoretical studies are based mostly on strictly one-dimensional (1D) models ${ }^{7-16}$ using effective Hamiltonians for description of the spin dynamics, although twodimensional ${ }^{6,18-21}$ approaches are also applied.

Most of the studies on SO interaction in quantum rings dealt with the pure Rashba interaction ${ }^{6-14,18-20}$ with neglected contribution of the Dresselhaus effect. Nevertheless, in III-V structures usually both SO coupling effects are present. Moreover, the case where both types of SO interaction have similar strength is particularly interesting: (i) for ballistic spin transport, ${ }^{23}$ (ii) for the formation of persistent spin helix in a two-dimensional electron gas, ${ }^{24,25}$ (iii) restoration of the isotropy of the spin exchange in double quantum dots, ${ }^{26}$ (iv) singlet-triplet avoided crossing in quantum dots, ${ }^{27}$ (v) tuning the amplitude of the persistent current oscillations excited by terahertz pulses, ${ }^{28}$ and (vi) deformation of the charge density in circular quantum rings. ${ }^{16,17}$

1D ring modeling for a pure Rashba coupling is based on an effective Hamiltonian, ${ }^{13}$ whose Hermitian form ${ }^{14}$ is taken by averaging the two-dimensional energy operator over the lowest state of the radial quantization. Similar operation was performed for the treatment of rings when both SO coupling types are present. ${ }^{15,16}$ In Ref. 17 it was found from a numerical diagonalization that for equal Rashba and Dresselhaus coupling constants the lowest-radial state approximation produces results that qualitatively differ from the exact ones in both the energy spectrum and the confined charge densities, and that-quite counterintuitively-these differences pertain for arbitrary small width of the ring. In this work, we develop an analytical matrix form for the model Hamiltonian that accounts for both the lowest and the first excited radial states. For a single type of SO coupling the present model, when limited to the lowest radial state, reproduces the formula of Frustaglia and Richter $^{29}$ for the energy spectrum. The lowest-radial-state approximation is qualitatively correct as long as a single type of SO coupling is present..$^{6-14,18-20}$ We demonstrate that when both Rashba and Dresselhaus SO interactions are present, this model needs to be extended to the two lowest radial states in order to correctly reproduce the features of the exact eigenstates. An analogy of this problem for quantum wires is also discussed.

The paper is organized as follows. In Sec. II we present the Hamiltonian model for both quantum ring and quantum wire and derive analytically an effective Hamiltonian matrix form for each case by taking into account the lowest and the first excited states. In Sec. III we give and discuss the results. Finally, a brief conclusion is given in Sec. IV.

\section{THEORY}

We investigate separately two quantum structure types in the presence of SO interactions: closed quantum rings and open quantum wires. Both the structures are confined within (001) and the magnetic field $\mathbf{B}=B \hat{\mathbf{e}}_{z}$ is oriented parallel to the growth [001] direction. The general form of the Hamiltonian is

$$
H_{0}=\left(\frac{\mathbf{P}^{2}}{2 m^{*}}+V(x, y)\right) \mathbf{1}+\frac{1}{2} g B \mu_{B} \sigma_{z}+H_{R}+H_{D},
$$

where $\mathbf{P}=-i \hbar \vec{\nabla}+e \mathbf{A}$ (A is the magnetic vector potential), $m^{*}$ is the electron effective mass, $V(x, y)$ is the confinement potential, $g$ represents the Landé factor, $\mu_{B}$ is the Bohr magneton, and $\sigma_{i}(i=x, y, z)$ stands for the $i$ th component of the Pauli matrices vector. $H_{R}$ and $H_{D}$ denote the linear Rashba and Dresselhaus SO coupling terms, respectively. To derive the effective Hamiltonian matrix for each case, we assume a parabolic profile for the confinement potential $V(x, y)$. 


\section{A. Effective Hamiltonian for quantum ring}

In this case it is convenient to represent the Hamiltonian in polar coordinates. Using the symmetric gauge $\mathbf{A}=\mathrm{Br} / 2 \hat{\mathbf{e}}_{\varphi}$ we have

$$
\mathbf{P}=-i \hbar \frac{\partial}{\partial r} \hat{\mathbf{e}}_{r}-i \frac{\hbar}{r}\left(\frac{\partial}{\partial \varphi}+i \frac{B \pi r^{2}}{\phi_{0}}\right) \hat{\mathbf{e}}_{\varphi} .
$$

The SO coupling terms take the form

$$
\begin{aligned}
H_{R}= & \frac{\alpha}{\hbar}\left(p_{y} \sigma_{x}-p_{x} \sigma_{y}\right)=i \alpha\left(-\sin \varphi \sigma_{x}+\cos \varphi \sigma_{y}\right) \frac{\partial}{\partial r} \\
& -\frac{i \alpha}{r}\left(\cos \varphi \sigma_{x}+\sin \varphi \sigma_{y}\right)\left(\frac{\partial}{\partial \varphi}+i \frac{B \pi r^{2}}{\phi_{0}}\right),
\end{aligned}
$$

and

$$
\begin{aligned}
H_{D}= & \frac{\beta}{\hbar}\left(p_{x} \sigma_{x}-p_{y} \sigma_{y}\right)=i \beta\left(-\cos \varphi \sigma_{x}+\sin \varphi \sigma_{y}\right) \frac{\partial}{\partial r} \\
& +\frac{i \beta}{r}\left(\sin \varphi \sigma_{x}+\cos \varphi \sigma_{y}\right)\left(\frac{\partial}{\partial \varphi}+i \frac{B \pi r^{2}}{\phi_{0}}\right),
\end{aligned}
$$

where $\phi_{0}=\frac{h}{e}$ is the flux quantum and $\alpha$ and $\beta$ are coupling constants. To derive a Hermitian expression for the radial part of the Hamiltonian, we consider the associated wave functions of $H_{0}$ as $\psi_{0}=r^{-\frac{1}{2}} \psi$. We then obtain

$$
\begin{aligned}
\left\langle H_{0}\right\rangle & =\iint \psi_{0}^{*}\left(H_{0} \psi_{0}\right) r d r d \varphi=\iint \psi^{*}\left(H^{r} \psi\right) d r d \varphi \\
& =\left\langle H^{r}\right\rangle
\end{aligned}
$$

where $H^{r}$ is a $2 \times 2$ matrix with the elements that, in the operator form, are as follows:

$$
\begin{gathered}
H_{11}^{r}=-\frac{\hbar^{2}}{2 m^{*}}\left[\frac{\partial^{2}}{\partial r^{2}}+\frac{1}{4 r^{2}}-\frac{1}{r^{2}}\left(i \frac{\partial}{\partial \varphi}-\frac{B \pi r^{2}}{\phi_{0}}\right)^{2}\right] \\
+V(r)+\frac{1}{2} g B \mu_{B} \\
H_{22}^{r}=H_{11}^{r}-g B \mu_{B} \\
H_{12}^{r}=-\frac{1}{r}\left(\alpha e^{-i \varphi}+i \beta e^{i \varphi}\right)\left(i \frac{\partial}{\partial \varphi}-\frac{B \pi r^{2}}{\phi_{0}}\right) \\
+\left(\alpha e^{-i \varphi}-i \beta e^{i \varphi}\right)\left(-\frac{1}{2 r}+\frac{\partial}{\partial r}\right)
\end{gathered}
$$

and

$$
\begin{aligned}
H_{21}^{r}= & \frac{1}{r}\left(-\alpha e^{i \varphi}+i \beta e^{-i \varphi}\right)\left(i \frac{\partial}{\partial \varphi}-\frac{B \pi r^{2}}{\phi_{0}}\right) \\
& -\left(\alpha e^{i \varphi}+i \beta e^{-i \varphi}\right)\left(-\frac{1}{2 r}+\frac{\partial}{\partial r}\right) .
\end{aligned}
$$

In Eq. (6), $V(r)=\frac{1}{2} m^{*} \omega_{r}^{2}\left(r-r_{0}\right)^{2}$ is the symmetric parabolic confinement potential where $\omega_{r}$ determines the effective width of the ring around the average radius $r_{0}$. The eigenfunctions of $H^{r}$ can be split as the sum of two spin-polarized spatial wave functions,

$$
\psi=\psi_{\uparrow} \chi_{+}+\psi_{\downarrow} \chi_{-}
$$

where $\chi_{+}$and $\chi_{-}$are the $\sigma_{z}$ eigenfunctions,

$$
\psi_{\uparrow}=\frac{1}{\sqrt{2 \pi}} \sum_{n, l} a_{n, l, \uparrow} e^{i l \varphi} R_{n l}(r),
$$

and

$$
\psi_{\downarrow}=\frac{1}{\sqrt{2 \pi}} \sum_{n, l} a_{n, l, \downarrow} e^{i l \varphi} R_{n l}(r),
$$

are the totally polarized spatial wave functions with spin-up and spin-down, respectively. In Eqs. (11) and (12), $l$ is the orbital angular momentum quantum number and $R_{n, l}(r)$ are the radial wave functions.

Given the operator form of $H^{r}$, we now turn to derive its analytical matrix representation. First, let us define the following parameters to make expressions as compact as possible: $a_{1} \equiv m^{*} \omega_{r} / \hbar, a_{2} \equiv B \pi / \phi_{0}, a_{3} \equiv \sqrt{a_{1}^{2}+a_{2}^{2}}$, and $a_{4} \equiv a_{1}^{2} r_{0} / a_{3}^{2}$. Using this definition we readily obtain the radial part of $H_{11}^{r}$ as

$$
\begin{aligned}
e^{-i l \varphi} H_{11}^{r} e^{i l \varphi}= & \frac{\hbar^{2}}{2 m^{*}}\left(-\frac{\partial^{2}}{\partial r^{2}}+\frac{l^{2}-\frac{1}{4}}{r^{2}}+a_{3}^{2}\left(r-a_{4}\right)^{2}\right. \\
& \left.+2 a_{2} l+a_{2}^{2} a_{4} r_{0}+\frac{m^{*} g B \mu_{B}}{\hbar^{2}}\right)
\end{aligned}
$$

The last two terms in Eq. (13) are independent of both $r$ and $l$. For sufficient large value of $\omega_{r}$, it is possible to find a cutoff radius $r_{c} \ll a_{4}$ so that the Gaussian distribution $e^{-a_{3}\left(r-a_{4}\right)^{2} / 2}$ virtually drops to zero at $r=r_{c}$. Then, for $r>r_{c}$ the term $\left(l^{2}-1 / 4\right) / r^{2}$ is a very slow-varying function compared to the parabolic term $a_{3}^{2}\left(r-a_{4}\right)^{2}$, so that the radial functions become independent of $l: R_{n l}(r)=R_{n}(r)$. Under these conditions, the normalized wave functions of the ground and first radial states can be accurately approximated by (see the Appendix)

$$
R_{0}=\left(\frac{a_{3}}{\pi}\right)^{\frac{1}{4}} e^{-\frac{a_{3}}{2}\left(r-a_{4}\right)^{2}}
$$

and

$$
R_{1}=\left(\frac{4 a_{3}^{3}}{\pi}\right)^{\frac{1}{4}}\left(r-a_{4}\right) e^{-\frac{a_{3}}{2}\left(r-a_{4}\right)^{2}},
$$

respectively. In the following we ignore the terms $R_{n}$ for $n>1$, since they correspond to energies that are too high to contribute to the low-energy part of the electron spectrum. Using the basis introduced in Eqs. (14) and (15), and noting that $\left\langle n^{\prime}, l^{\prime}\left|H_{i j}^{r}\right| n, l\right\rangle \equiv \int_{0}^{\infty} \int_{0}^{2 \pi} R_{n^{\prime}}^{*} e^{-i l^{\prime} \varphi} H_{i j}^{r} R_{n} e^{i l \varphi} d \varphi d r$, we find (see the Appendix)

$$
\begin{aligned}
\left\langle n^{\prime}, l^{\prime}\left|H_{11}^{r}\right| n, l\right\rangle= & \frac{\hbar^{2}}{2 m^{*}} \delta_{n^{\prime}, n} \delta_{l^{\prime}, l}\left(a_{3}(2 n+1)+\frac{\left(l^{2}-\frac{1}{4}\right)}{a_{4}^{2}}\right. \\
& \left.+2 a_{2} l+a_{2}^{2} a_{4} r_{0}+\frac{m^{*} g B \mu_{B}}{\hbar^{2}}\right) \\
\left\langle n^{\prime}, l^{\prime}\left|H_{22}^{r}\right| n, l\right\rangle= & \left\langle n^{\prime}, l^{\prime}\left|H_{11}^{r}\right| n, l\right\rangle-g B \mu_{B} \delta_{l^{\prime}, l} \delta_{n^{\prime}, n},
\end{aligned}
$$

$$
\begin{aligned}
& \left\langle n^{\prime}, l^{\prime}\left|H_{12}^{r}\right| n, l\right\rangle \\
& =\left[\left(\frac{l}{a_{4}}+a_{2} a_{4}\right) \delta_{n^{\prime}, n}+\frac{a_{2}}{\sqrt{2 a_{3}}} \delta_{n^{\prime}, n \pm 1}\right]\left(\alpha \delta_{l^{\prime}, l-1}+i \beta \delta_{l^{\prime}, l+1}\right) \\
& \quad+\left(-\frac{\delta_{n^{\prime}, n}}{2 a_{4}}+\sqrt{\frac{a_{3}}{2}}\left(\delta_{n^{\prime}, n-1}-\delta_{n^{\prime}, n+1}\right)\right) \\
& \quad \times\left(\alpha \delta_{l^{\prime}, l-1}-i \beta \delta_{l^{\prime}, l+1}\right),
\end{aligned}
$$


and also

$$
\begin{aligned}
\left\langle n^{\prime}, l^{\prime}\left|H_{21}^{r}\right| n, l\right\rangle= & {\left[\left(\frac{l}{a_{4}}+a_{2} a_{4}\right) \delta_{n^{\prime}, n}+\frac{a_{2}}{\sqrt{2 a_{3}}} \delta_{n^{\prime}, n \pm 1}\right]\left(\alpha \delta_{l^{\prime}, l+1}-i \beta \delta_{l^{\prime}, l-1}\right) } \\
& -\left(-\frac{\delta_{n^{\prime}, n}}{2 a_{4}}+\sqrt{\frac{a_{3}}{2}}\left(\delta_{n^{\prime}, n-1}-\delta_{n^{\prime}, n+1}\right)\right)\left(\alpha \delta_{l^{\prime}, l+1}+i \beta \delta_{l^{\prime}, l-1}\right),
\end{aligned}
$$

where $n^{\prime}$ and $n$ can take the values 0 or 1 .

Based on Eqs. (16)-(19), we introduce a Hamiltonian whose matrix components are each equivalent to a $4 \times 4$ matrix including the effects of the two lowest radial states in addition to the spin

$$
H^{r}\left(l^{\prime}, l\right) \equiv\left\langle l^{\prime}\left|H^{r}\right| l\right\rangle=\left(\begin{array}{cccc}
\left\langle 0, l^{\prime}\left|H_{11}^{r}\right| 0, l\right\rangle & 0 & \left\langle 0, l^{\prime}\left|H_{12}^{r}\right| 0, l\right\rangle & \left\langle 0, l^{\prime}\left|H_{12}^{r}\right| 1, l\right\rangle \\
0 & \left\langle 1, l^{\prime}\left|H_{11}^{r}\right| 1, l\right\rangle & \left\langle 1, l^{\prime}\left|H_{12}^{r}\right| 0, l\right\rangle & \left\langle 1, l^{\prime}\left|H_{12}^{r}\right| 1, l\right\rangle \\
\left\langle 0, l^{\prime}\left|H_{21}^{r}\right| 0, l\right\rangle & \left\langle 0, l^{\prime}\left|H_{21}^{r}\right| 1, l\right\rangle & \left\langle 0, l^{\prime}\left|H_{22}^{r}\right| 0, l\right\rangle & 0 \\
\left\langle 1, l^{\prime}\left|H_{21}^{r}\right| 0, l\right\rangle & \left\langle 1, l^{\prime}\left|H_{21}^{r}\right| 1, l\right\rangle & 0 & \left\langle 1, l^{\prime}\left|H_{22}^{r}\right| 1, l\right\rangle
\end{array}\right) .
$$

Two ranks of the above $4 \times 4$ matrix are due to the ground and first radial states and the others come from the possible spin states (spin-up and spin-down). Accordingly, each element in the corresponding eigenvectors of $H^{r}$ correspond to a fourcomponent vector as $\left[a_{0, l, \uparrow} a_{1, l, \uparrow} a_{0, l, \downarrow} a_{1, l, \downarrow}\right]^{T}$.

\section{Relation to lowest-radial-state approximation and Richter-Frustaglia limit}

To approach the routine lowest-radial-state approximation for a strictly $1 \mathrm{D}$ ring one sets $n^{\prime}=n=0$ which reduces $H^{r}\left(l^{\prime}, l\right)$ in Eq. (20) into a $2 \times 2$ matrix. Then it is enough to consider the limit $\omega_{r} \rightarrow \infty$ and exclude the terms diverging in Eqs. (16)-(19); namely $a_{3}(2 n+1)$ in Eq. (16). In addition, it is obvious that $r=r_{0}, a_{4}=r_{0}, a_{2}=\phi / r_{0}^{2} \phi_{0}$ ( $\phi$ is the magnetic flux threading the ring), and also $\delta_{n^{\prime}, n \pm 1}=0$. By fixing the quantum number $n=0$ we obtain the angle-dependent components of the $2 \times 2$ Hamiltonian,

$$
\begin{aligned}
\left\langle l^{\prime}\left|H_{11}^{r}\right| l\right\rangle= & \frac{\hbar^{2}}{2 m^{*} r_{0}^{2}} \delta_{l^{\prime}, l}\left(l+\frac{\phi}{\phi_{0}}\right)^{2}+\frac{1}{2} \delta_{l^{\prime}, l} g B \mu_{B}, \\
\left\langle l^{\prime}\left|H_{22}^{r}\right| l\right\rangle=\left\langle l^{\prime}\left|H_{11}^{r}\right| l\right\rangle-\delta_{l^{\prime}, l} g B \mu_{B}, & \\
\left\langle l^{\prime}\left|H_{12}^{r}\right| l\right\rangle= & \left(l+\frac{\phi}{\phi_{0}}\right)\left(\frac{\alpha}{r_{0}} \delta_{l^{\prime}, l-1}+i \frac{\beta}{r_{0}} \delta_{l^{\prime}, l+1}\right) \\
& -\frac{1}{2}\left(\frac{\alpha}{r_{0}} \delta_{l^{\prime}, l-1}-i \frac{\beta}{r_{0}} \delta_{l^{\prime}, l+1}\right) \\
\left\langle l^{\prime}\left|H_{21}^{r}\right| l\right\rangle= & \left(l+\frac{\phi}{\phi_{0}}\right)\left(\frac{\alpha}{r_{0}} \delta_{l^{\prime}, l+1}-i \frac{\beta}{r_{0}} \delta_{l^{\prime}, l-1}\right) \\
& +\frac{1}{2}\left(\frac{\alpha}{r_{0}} \delta_{l^{\prime}, l+1}+i \frac{\beta}{r_{0}} \delta_{l^{\prime}, l-1}\right) .
\end{aligned}
$$

In Eq. (21) we ignored the constant term $\hbar^{2} / 8 m^{*} r_{0}^{2}$ that does not depend on $l$.

Now consider a case when only the Rashba SO coupling is present; i.e., $\beta=0$ and $g=0$. Using Eqs. (21)-(24), the eigenvalues can be obtained from the below eigenvalue equation:

$$
\begin{aligned}
& \left(\begin{array}{cc}
\frac{\hbar^{2}}{2 m^{*} r_{0}^{2}}\left(l+\frac{\phi}{\phi_{0}}\right)^{2} & \frac{\alpha}{r_{0}}\left(l+\frac{\phi}{\phi_{0}}+\frac{1}{2}\right) \\
\frac{\alpha}{r_{0}}\left(l+\frac{\phi}{\phi_{0}}+\frac{1}{2}\right) & \frac{\hbar^{2}}{2 m^{*} r_{0}^{2}}\left(l+\frac{\phi}{\phi_{0}}+1\right)^{2}
\end{array}\right)\left(\begin{array}{c}
a_{l, \uparrow} \\
a_{l+1, \downarrow}
\end{array}\right) \\
& =E_{R}\left(\begin{array}{c}
a_{l, \uparrow} \\
a_{l+1, \downarrow}
\end{array}\right),
\end{aligned}
$$

which yields the eigenvalues previously derived by RichterFrustaglia, ${ }^{29}$

$$
\begin{aligned}
E_{R}= & \frac{\hbar^{2}}{2 m^{*} r_{0}^{2}}\left[\frac{1}{4}+\left(l+\frac{\phi}{\phi_{0}}+\frac{1}{2}\right)^{2}\right. \\
& \left. \pm\left(l+\frac{\phi}{\phi_{0}}+\frac{1}{2}\right) \sqrt{1+\left(\frac{2 \alpha m^{*} r_{0}}{\hbar^{2}}\right)^{2}}\right] .
\end{aligned}
$$

\section{B. Effective Hamiltonian for quantum wire}

We consider a quantum wire that is confined parabolically in [010] direction by the potential $V(y)=\frac{1}{2} m^{*} \omega_{w}^{2} y^{2}$. The wire is aligned along the $x$ axis and the potential is independent of $x$ component. Therefore, the overall wave function has a definite wave number $k_{x}$ along the wire so that the relevant spin-polarized spatial wave functions can be written as

$$
\phi_{\uparrow(\downarrow)}=e^{i k_{x} x} \sum_{n} b_{n, \uparrow(\downarrow)} \phi_{n}(y),
$$

where $\phi_{n}(y)$ is the wave function in $y$ direction. Using the Coulomb gauge $\mathbf{A}=(-B y, 0,0)$ the Hamiltonian in Eq. (1) reads

$$
\begin{aligned}
H_{0}= & \frac{\hbar^{2} k_{x}^{2}}{2 m^{*}}\left(1-\frac{\omega_{c}^{2}}{\Omega^{2}}\right) \mathbf{1}+\frac{p_{y}^{2}}{2 m^{*}} \mathbf{1}+\frac{1}{2} m^{*} \Omega^{2}\left(y-y_{0}\right)^{2} \mathbf{1} \\
& +\alpha\left(\frac{p_{y}}{\hbar} \sigma_{x}-k_{x} \sigma_{y}\right)+\beta\left(k_{x} \sigma_{x}-\frac{p_{y}}{\hbar} \sigma_{y}\right)+\frac{1}{2} g B \mu_{B} \sigma_{z},
\end{aligned}
$$

where $\omega_{c}=e B / m^{*}$ is the cyclotron frequency, $\Omega=$ $\sqrt{\omega_{c}^{2}+\omega_{w}^{2}}$ is the effective confinement strength due to the parabolic potential and the magnetic field, and $y_{0}=$ 
$\hbar \omega_{c} k_{x} / m^{*} \Omega^{2}$. The momentum operator $p_{y}$ can be written in terms of the standard ladder operators $a_{y}$ and $a_{y}^{\dagger}$ as $p_{y}=i \sqrt{m^{*} \Omega \hbar / 2}\left(a_{y}^{\dagger}-a_{y}\right)$. Then, in the basis of the occupation number operator $\hat{N}=a_{y}^{\dagger} a_{y}$ [i.e., $\phi_{n}(y)=|n\rangle$, where $n=0,1,2, \ldots]$, we obtain the matrix representation of the Hamiltonian components,

$$
\begin{aligned}
&\left\langle n^{\prime}\left|H_{11}^{w}\right| n\right\rangle= \delta_{n, n^{\prime}}\left[\frac{\hbar^{2} k_{x}^{2}}{2 m^{*}}\left(1-\frac{\omega_{c}^{2}}{\Omega^{2}}\right)+\hbar \Omega\left(n+\frac{1}{2}\right)\right. \\
&+\left.\frac{g B \mu_{B}}{2}\right], \\
&\left\langle n^{\prime}\left|H_{22}^{w}\right| n\right\rangle=\left\langle n^{\prime}\left|H_{11}^{w}\right| n\right\rangle-g B \mu_{B} \delta_{n, n^{\prime}}, \\
&\left\langle n^{\prime}\left|H_{12}^{w}\right| n\right\rangle=k_{x} \delta_{n, n^{\prime}}(\beta+i \alpha)+\sqrt{\frac{m^{*} \Omega}{2 \hbar}}(-\beta+i \alpha) \\
& \times\left(\sqrt{n+1} \delta_{n^{\prime}, n+1}-\sqrt{n} \delta_{n^{\prime}, n-1}\right),
\end{aligned}
$$

and

$$
\begin{aligned}
\left\langle n^{\prime}\left|H_{21}^{w}\right| n\right\rangle= & k_{x} \delta_{n, n^{\prime}}(\beta-i \alpha)+\sqrt{\frac{m^{*} \Omega}{2 \hbar}}(\beta+i \alpha) \\
& \times\left(\sqrt{n+1} \delta_{n^{\prime}, n+1}-\sqrt{n} \delta_{n^{\prime}, n-1}\right) .
\end{aligned}
$$

To approach the lowest sub-band approximation one sets $n^{\prime}=n=0$. This yields a $2 \times 2$ Hamiltonian matrix whose eigenenergies are given by

$$
\begin{aligned}
E_{0}\left(k_{x}\right)= & \frac{\hbar^{2} k_{x}^{2}}{2 m^{*}}\left(1-\frac{\omega_{c}^{2}}{\Omega^{2}}\right)+\frac{1}{2} \hbar \Omega \\
& \pm \sqrt{\frac{1}{4} g^{2} B^{2} \mu_{B}^{2}+k_{x}^{2}\left(\alpha^{2}+\beta^{2}\right)}
\end{aligned}
$$

Note that for a strictly 1D quantum wire it is enough to consider the limit $\omega_{w} \rightarrow \infty$ and remove the terms diverging in Eq. (33).

Now let us consider the effects of the first excited sub-band in addition to the lowest one. In this case we attain a $4 \times 4$ Hamiltonian matrix similar to a one presented in Eq. (20). By diagonalizing the $4 \times 4$ Hamiltonian matrix one obtains the eigenenergies as

$$
\begin{aligned}
& E\left(k_{x}\right) \\
& =\frac{\hbar^{2} k_{x}^{2}}{2 m^{*}}\left(1-\frac{\omega_{c}^{2}}{\Omega^{2}}\right)+\hbar \Omega \\
& \quad \pm \frac{1}{2} \sqrt{\hbar^{2} \Omega^{2}+g^{2} B^{2} \mu_{B}^{2}+4\left(\frac{m^{*} \Omega}{2 \hbar}+k_{x}^{2}\right)\left(\alpha^{2}+\beta^{2}\right) \pm 4 \gamma}
\end{aligned}
$$

where

$$
\gamma=\sqrt{k_{x}^{2} \hbar^{2} \Omega^{2}\left(\alpha^{2}+\beta^{2}\right)+\frac{8}{\hbar} m^{*} \Omega k_{x}^{2} \alpha^{2} \beta^{2}+\frac{\left(\hbar \Omega g B \mu_{B}\right)^{2}}{4}} .
$$

Based on the fact that under a unitary transformation the energy eigenvalues remain unchanged, we introduce the unitary operator

$$
u=\left(\begin{array}{cc}
0 & \sqrt{i} \\
\sqrt{-i} & 0
\end{array}\right)
$$

so that $u^{\dagger} \sigma_{x} u=-\sigma_{y}, u^{\dagger} \sigma_{y} u=-\sigma_{x}$, and $u^{\dagger} \sigma_{z} u=\sigma_{z}$. Using these relations we find from Eq. (28) that $u^{\dagger} H_{0}(\alpha, \beta) u=$ $H_{0}(\beta, \alpha)$ or, in other words, $E(\alpha, \beta)=E(\beta, \alpha)$. This means that by exchanging the SO couplings strengths the energy remains unchanged, which is obvious in Eqs. (33) and (34).

\section{RESULTS AND DISCUSSION}

For our calculations we consider the parameters corresponding to etched InGaAs/GaAs materials ${ }^{30}: m^{*}=0.063 m_{e}$ and-unless stated otherwise $-\beta=10.8 \mathrm{meV} \mathrm{nm}$. This value is obtained from the bulk Dresselhaus constant $\left(\beta_{b}\right)$ as $\beta=$ $\frac{\pi}{d} \beta_{b}$, where $d=5 \mathrm{~nm}$ is the height of the structure in the growth direction and $\beta_{b}=27.5 \mathrm{eV} \AA^{3}$ for GaAs. ${ }^{31} \mathrm{We}$ set $g=-2.15$ when the Zeeman effect is present.

Figure 1 shows the energy spectrum as a function of the $B$ for a quantum ring with $r_{0}=50 \mathrm{~nm}$ and $\hbar \omega_{r}=30 \mathrm{meV}$. The panels are for various values of the Rashba constant $\alpha$ and $g$. The spectrum without SO coupling was plotted in Fig. 1(a) by the blue dashed lines. The rest of the plots show the results in the presence of SO coupling: the red dotted curves correspond to the energies obtained in the lowest-radial-state approximation and the solid curves indicate those for which the first-excited radial state is also taken into account. The results of the lowest-radial-state approximation are shifted up by the constant energy $\hbar^{2}\left(a_{1}+0.25 / r_{0}^{2}\right) / 2 m^{*}=15.06 \mathrm{meV}$ in order that the results became comparable to the other plots.

At the lower half of each plot, the lines referred to the right axis indicate the average value of the total ground-state angular momentum. The discontinuous drops in the value of $\left\langle J_{z}\right\rangle$ correspond to the ground-state angular momentum transitions. In Fig. 1(a) the value of $\left\langle J_{z}\right\rangle$ is illustrated in terms of infinitesimal deviations $\pm \delta \beta$ because the energies are twofold spin degenerate. Notice that the stepwise variations of $J_{z}$ occur twice more often than those of nondegenerate states shown in Fig. 1(b); half of them which take place simultaneously with the ground-state level crossings are due to total angular momentum transitions (both the spin and the angular momentum change) and the other half are due to only spin transitions.

The case of equal coupling constants $\alpha=\beta$ and $g=0$ is very special in quantum rings. In this case the entire energy spectrum in the absence of SO coupling is expected to be shifted by $\Delta \mathrm{SO}=-2 \alpha^{2} m^{*} / \hbar^{2}$ down in order to reproduce the energy spectrum with $\mathrm{SO}$ interactions. ${ }^{23,32}$ The value of $\triangle \mathrm{SO}$ in Fig. 1(a) is just equal to $-2 \alpha^{2} m^{*} / \hbar^{2}=$ $-0.192 \mathrm{meV}$ for the case with included first excited radial state. Moreover, for $\alpha=\beta$ and $g=0$ the Hamiltonian commutes with the operator of the [1]10] spin component. ${ }^{23,32}$ The Hamiltonian eigenfunctions $\psi_{ \pm}$corresponding to the $\pm \hbar / 2$ spin eigenvalues in the [1 $\overline{10}$ ] direction are related to the eigefunctions $\varphi$ obtained in the absence of SO coupling as

$$
\psi_{ \pm}(x, y)=\frac{1}{\sqrt{2}}\left(\begin{array}{c}
1 \\
\pm e^{-i \pi / 4}
\end{array}\right) \varphi(x, y) e^{\mp \frac{i \sqrt{2} \alpha m^{*}}{\hbar^{2}}(x+y)} .
$$

Since $|\varphi|^{2}$ is circularly symmetric, therefore the charge densities for the eigenstates $\psi_{ \pm}$are also circularly symmetric. 

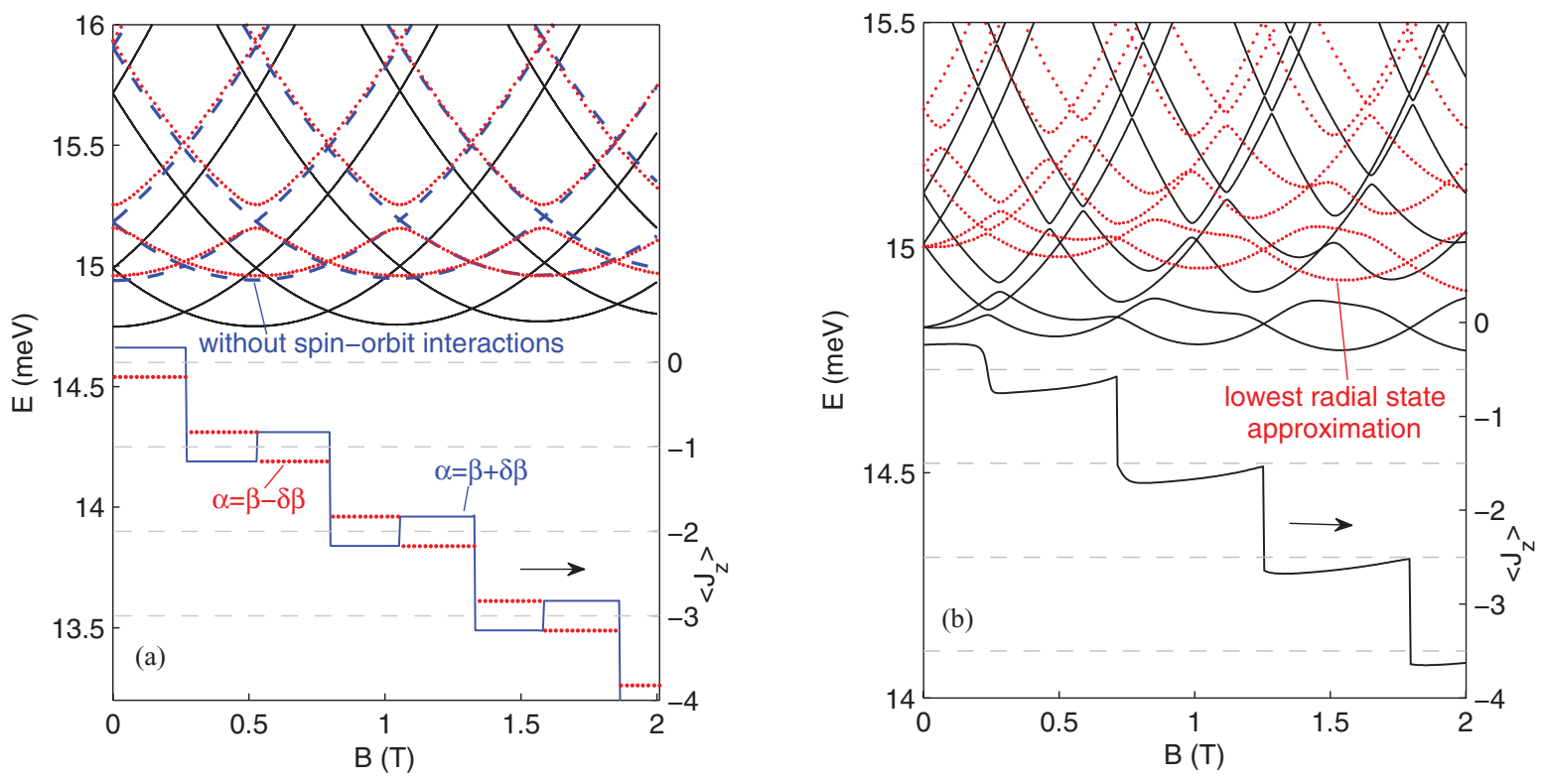

FIG. 1. (Color online) Upper curves show the energy spectrum versus the magnetic field for $\alpha=\beta, g=0$ (a) and for $\alpha=\beta / 2=5.4 \mathrm{meV}$ $\mathrm{nm}, g=-2.15$ (b). The red dotted curves indicate the results of the lowest-radial-state approximation $(n=0)$ that are shifted up by $15.06 \mathrm{meV}$. The solid curves are obtained using the new derived Hamiltonian in which the first-excited radial state is included as well as the ground-radial state $(n=0,1)$. The dashed blue curves in panel (a) indicate the energy spectrum without SO interactions. The lower lines, which are referred to the right axis, display the average value measured over the $z$ component of the ground-state total angular momentum. In panel (a), the value $\left\langle J_{z}\right\rangle$ is shown for two infinitesimal changes in the value of $\alpha$ as much as $\pm \delta \beta= \pm 10^{-10} \beta$ because the energy spectrum is twofold spin degenerate.

The charge density as obtained with considering both $n=0$ and $n=1$ radial states is shown in Fig. 2(b) (left panel). It is seen that the charge density is circularly symmetric. In fact, the calculated density is circular with a precision of four significant digits.

On the other hand, when one restricts the basis to the lowest radial state, one obtains (i) avoided crossings in the energy spectrum [see the red dotted curve in Fig. 1(a)], and (ii) the deviation of the charge density from circular symmetry-see Fig. 2(a) (left panel), which are artifacts of the applied approximation.

When the Zeeman effect is present [Fig. 1(b)], the energy spectrum of the lowest-radial state approximation are qualitatively similar to the exact one with similar avoided crossings.
Nevertheless, Fig. 2 shows that also for the case of $\alpha=\beta / 2$, $g=-2.15$, and $B=0.75 \mathrm{~T}$, the charge density obtained in the lowest radial approximation state differs qualitatively from the exact one: in the lowest-radial-state approximation the maximal charge density are oriented along the antidiagonal $(x=-y)$ which is in contrast to the result obtained for the basis with $n=0,1$.

The restriction of the basis to the lowest radial state when $\omega_{r}$ tends to $\infty$ seems natural, which calls for a comment on the origin of the qualitative error introduced by this step. Let us focus on the $\omega_{r} \rightarrow \infty$ and $B=0$ case. The energy difference of the diagonal matrix elements corresponding to the $n=0$ and $n=1$ radial state increases linearly with $\omega_{r}$ [see Eq. (16)]. The off-diagonal elements mixing the $n=1$ and $n=0$ states with
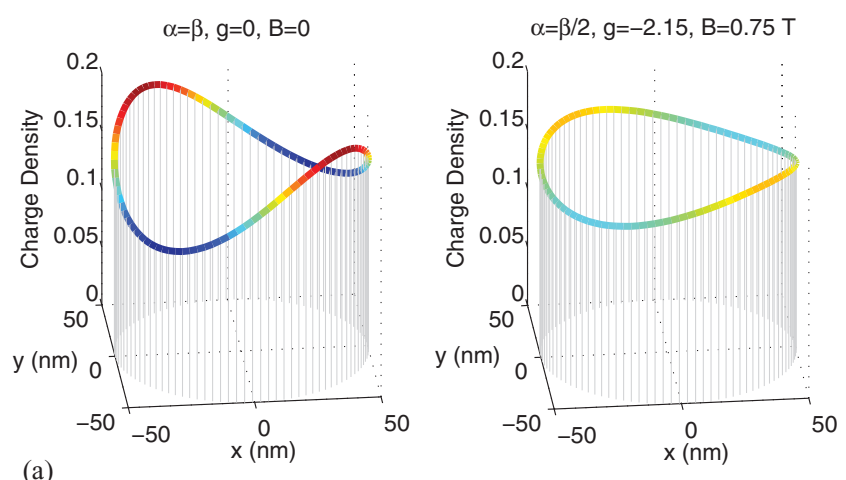

FIG. 2. (Color online) Charge density distributions obtained with the use of the lowest-radial-state approximation (a) and when the first-excited radial state is also included (b). 

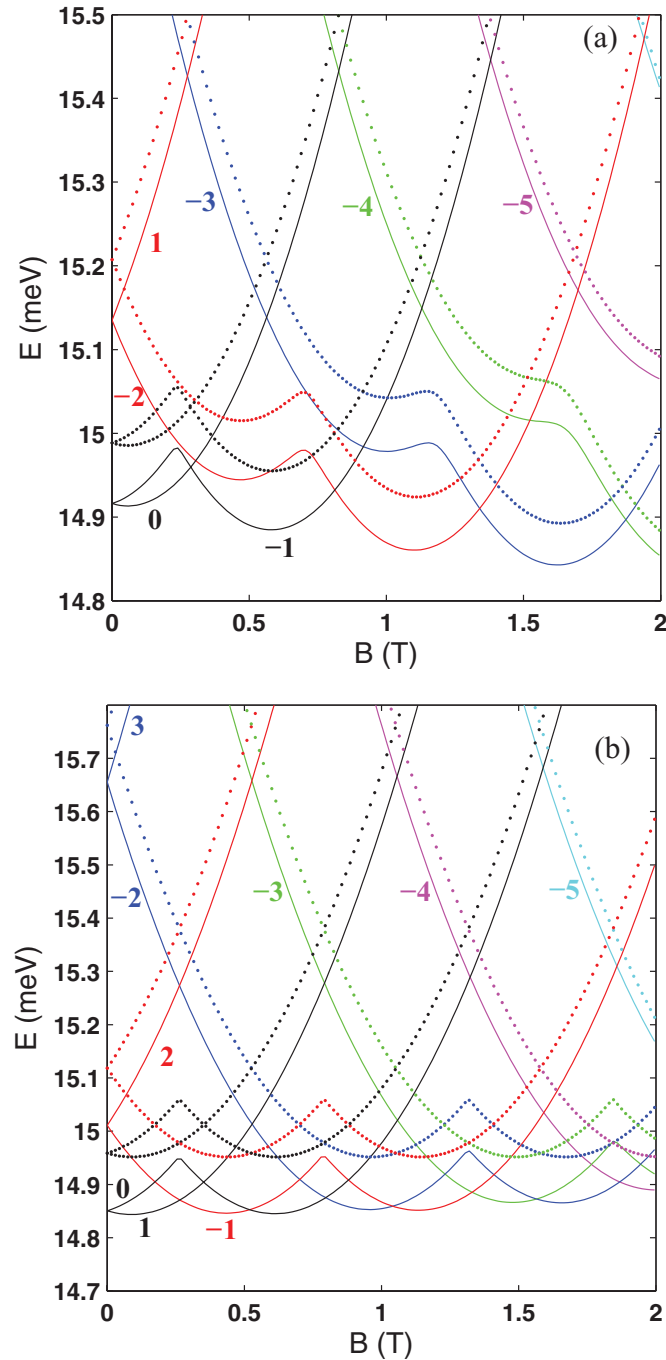

FIG. 3. (Color online) Energy spectrum versus the magnetic field in the presence of a single type of spin-orbit interaction for pure Rashba coupling with $\alpha=5.4 \mathrm{meV} \mathrm{nm}$ and $g=-2.15$ (a) and for pure Dresselhaus coupling with $g=0$ (b). The dotted curves show the energy spectrum obtained using the lowest-radial-state approximation and the solid curves show those in which the first excited state is also included. The numbers denote the values of the orbital angular momentum for the solid curves in $\hbar$ units.

adjacent values of $l$ number, increase as $\sqrt{\omega_{r}}$ [see Eqs. (18) and (19)]. Although it could seem that the off-diagonal terms might be neglected as compared to the diagonal ones, this is not the case. In order to demonstrate this we consider the matrix

$$
M=\left(\begin{array}{cc}
0 & \sqrt{\omega_{r}} \\
\sqrt{\omega_{r}} & \omega_{r}
\end{array}\right),
$$

with eigenvalues $\frac{\omega_{r}}{2} \pm \frac{1}{2} \sqrt{\omega_{r}^{2}+4 \omega_{r}}$. The lowest eigenvalue in the $\omega_{r} \rightarrow \infty$ limit tends to -1 and not 0 . The square-root increase of the off-diagonal elements compensates for the linear increase in the $M_{22}$ element. In the studied case, the $n=0$ approximation differs qualitatively from the exact results for the energy spectrum and the charge density, also
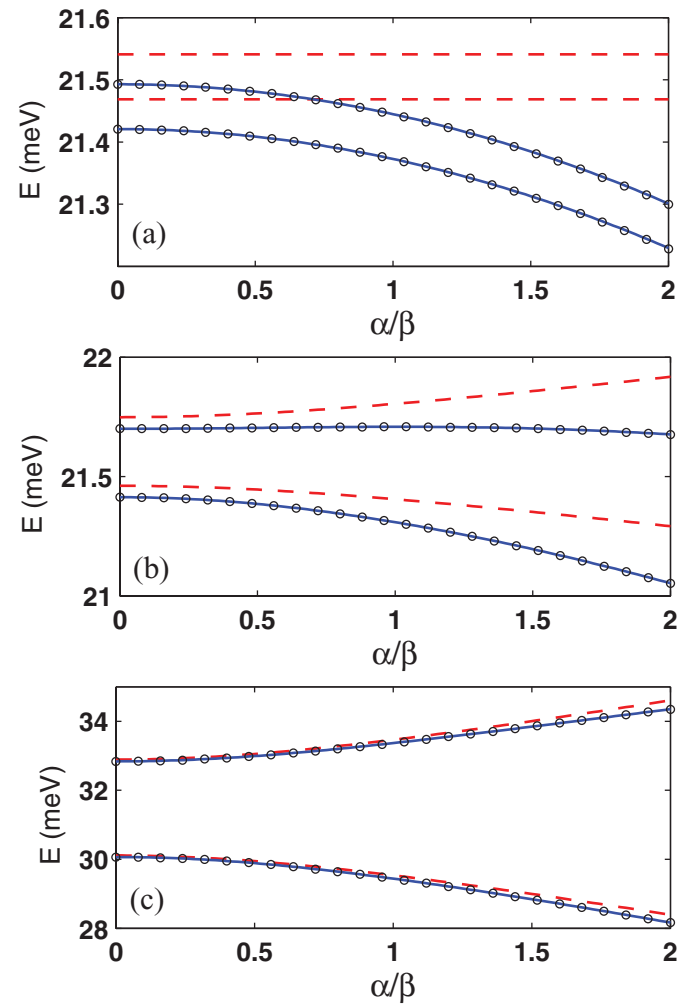

FIG. 4. (Color online) Two lowest eigenenergies of a quantum wire versus Rashba SO constant for $g=-2.5, \hbar \omega_{w}=43 \mathrm{meV}$, $B=0.5 \mathrm{~T}$ and (a) $k_{x}=0$, (b) $\hbar^{2} k_{x}^{2} / 2 m^{*}=0.1 \mathrm{meV}$, and (c) $\hbar^{2} k_{x}^{2} / 2 m^{*}=10 \mathrm{meV}$. The circles indicate the energies obtained by the exact numerical diagonalization. The dashed curves indicate the energies produced by the lowest sub-band approximation and the solid curves are for accounting the first excited sub-band in addition to the lowest one.

in the $\omega_{r} \rightarrow \infty$ limit. The linear increase of $\omega_{r}$ in the $n=1$ energy level is compensated by the $\sqrt{\omega_{r}}$ increase in the SO coupling elements. This clearly shows that one cannot indicate any value of $\omega_{r}$ large enough to justify the lowest-radial-state approximation.

The energy spectra of a quantum ring as obtained with both bases is given in Fig. 3 when only a single type of SO coupling is present. Each energy level in the spectrum corresponds to a definite orbital angular momentum $(l)$. At $B=0$, for the presence of pure Rashba coupling we have $E(-|l|)=E(|l|-$ 1) while for pure Dresselhaus coupling we have $E(-|l|)=$ $E(|l|+1)$. The figure reveals that the neglect of the $n=1$ state in the basis affects the energy levels, but with no qualitative consequences for the character of the spectrum.

Figure 4 displays two lowest eigenenergies of a quantum wire versus Rashba coupling constant for different wave numbers $k_{x}$. The magnetic field is $B=0.5 \mathrm{~T}$ and the confinement strength is $\hbar \omega_{w}=43 \mathrm{meV}$ corresponding to the wire width $W=\sqrt{8 \hbar / m^{*} \omega_{w}} \approx 15 \mathrm{~nm}$. The dashed curves indicate the energies obtained by the lowest sub-band approximation and the solid curves show those for which the first excited sub-band is taken into account besides the lowest one. The circles correspond to the exact eigenenergies calculated by the numerical diagonalization of $H_{0}$ in Eq. (28). The restriction 
of the basis to the lowest sub-band produces qualitatively incorrect results when the wave number $k_{x}$ is too small or, more generally, when $\hbar^{2} k_{x} / 2 m^{*} \ll \sqrt{\alpha^{2}+\beta^{2}}$. For $k_{x}=0$ the eigenenergies of the lowest sub-band approximation remain constant as the Rashba coupling strength is increased while the accounting of the second sub-band leads the eigenenergies to decrease [see Fig. 4(a)]. Similar deviation from the exact results are observed in Fig. 4(b) for $\hbar^{2} k_{x}^{2} / 2 m^{*}=0.1 \mathrm{meV}$. For the larger values of $k_{x}$ the results of the lowest subband approximation are in consistent with those obtained by considering both $n=0$ and $n=1$ sub-bands [see Fig. 4(c)].

\section{SUMMARY AND CONCLUSIONS}

We have considered an electron in a circular quantum ring with both the Rashba and Dresselhaus coupling constants. We assumed a parabolic profile for the confinement potential and derived an analytical matrix form of the effective Hamiltonian using radial eigenstates in the quasi-one-dimensional limit of large radius and large confinement energy. We have demonstrated that, in the presence of both SO couplings, it is sufficient to include up to the first excited radial state in order to correctly describe the qualitative features of the energy spectra and charge densities. We have explained why the basis limited to the lowest radial state remains an invalid approximation also in the strictly one-dimensional limit. On the other hand, our model supports the applicability of the lowest-radial-state approximation for the special case when only a single type of SO coupling is present, and it reproduces the classical formula ${ }^{29}$ for the energy spectrum.

\section{ACKNOWLEDGMENTS}

This work was partially supported by Polish Ministry of Science and Higher Education and its grants for Scientific Research.

\section{APPENDIX: SUPPLEMENTARY NOTES FOR DERIVATION OF EQS. (14)-(19)}

Recalling that the Gaussian function $e^{-a_{3}\left(r-a_{4}\right)^{2} / 2}$ is practically zero for $r \leqslant r_{c}$, we obtain

$$
\begin{aligned}
\int_{0}^{\infty} e^{-a_{3}\left(r-a_{4}\right)^{2}} d r & \simeq \int_{r_{c}}^{a_{4}} e^{-a_{3}\left(r-a_{4}\right)^{2}} d r+\int_{a_{4}}^{\infty} e^{-a_{3}\left(r-a_{4}\right)^{2}} d r \\
& =2 \int_{a_{4}}^{\infty} e^{-a_{3}\left(r-a_{4}\right)^{2}} d r=\sqrt{\frac{\pi}{a_{3}}}
\end{aligned}
$$

and similarly

$$
\begin{aligned}
& \int_{0}^{\infty}\left(r-a_{4}\right)^{2} e^{-a_{3}\left(r-a_{4}\right)^{2}} d r \\
& \quad \simeq 2 \int_{a_{4}}^{\infty}\left(r-a_{4}\right)^{2} e^{-a_{3}\left(r-a_{4}\right)^{2}} d r=\sqrt{\frac{\pi}{4 a_{3}^{3}}} .
\end{aligned}
$$

Note that if $r_{0}$ is not too small and the confinement potential is sufficiently sharp, the term $1 / r$ is a very slow-varying function with respect to the Gaussian weight function $e^{-a_{3}\left(r-a_{4}\right)^{2}}$ while $r>r_{c}$. Therefore, we have

$$
\begin{aligned}
& \int_{r_{c}}^{\infty} \frac{1}{r} e^{-a_{3}\left(r-a_{4}\right)^{2}} d r \\
& \quad \simeq \frac{1}{a_{4}} \int_{r_{c}}^{\infty} e^{-a_{3}\left(r-a_{4}\right)^{2}} d r=\frac{1}{a_{4}} \sqrt{\frac{\pi}{a_{3}}} .
\end{aligned}
$$

Continuing along the same lines of reasoning, we obtain

$$
\begin{gathered}
\int_{0}^{\infty} r R_{n^{\prime}}^{*} R_{n} d r=a_{4} \delta_{n^{\prime}, n}+\frac{1}{\sqrt{2 a_{3}}} \delta_{n^{\prime}, n \pm 1}, \\
\int_{0}^{\infty} \frac{1}{r} R_{n^{\prime}}^{*} R_{n} d r=\frac{1}{a_{4}} \delta_{n^{\prime}, n}, \\
\int_{0}^{\infty} R_{n^{\prime}}^{*} \frac{\partial}{\partial r} R_{n} d r=\sqrt{\frac{a_{3}}{2}}\left(\delta_{n^{\prime}, n-1}-\delta_{n^{\prime}, n+1}\right),
\end{gathered}
$$

where $n^{\prime}$ and $n$ can take the values 0 or 1 .

\footnotetext{
${ }^{1}$ Y. A. Bychkov and E. I. Rashba, J. Phys. C 17, 6039 (1984).

${ }^{2}$ G. Dresselhaus, Phys. Rev. 100, 580 (1955).

${ }^{3}$ D. Awschalom, D. Loss, and N. Samarth, Semiconductor Spintronics and Quantum Computation (Springer, Berlin, 2002).

${ }^{4} \mathrm{R}$. Winkler, Spin-Orbit Coupling Effects in Two-Dimensional Electron and Hole Systems (Springer, Berlin, 2003).

${ }^{5}$ J.-B. Yau, E. P. De Poortere, and M. Shayegan, Phys. Rev. Lett. 88, 146801 (2002); S. Souma and B. K. Nikolic, ibid. 94, 106602 (2005); M. J. van Veenhuizen, T. Koga, and J. Nitta, Phys. Rev. B 73, 235315 (2006); M. König, A. Tschetschetkin, E. M. Hankiewicz, J. Sinova, V. Hock, V. Daumer, M. Schäfer, C. R. Becker, H. Buhmann, and L. W. Molenkamp, Phys. Rev. Lett. 96, 076804 (2006).

${ }^{6}$ D. Frustaglia, M. Hentschel, and K. Richter, Phys. Rev. Lett. 87, 256602 (2001).

${ }^{7}$ P. Földi, O. Kalman, and F. M. Peeters, Phys. Rev. B 80, 125324 (2009).
}

${ }^{8}$ B. Molnar, F. M. Peeters, and P. Vasilopoulos, Phys. Rev. B 69, 155335 (2004).
${ }^{9}$ P. Földi, B. Molnar, M. G. Benedict, and F. M. Peeters, Phys. Rev. B 71, 033309 (2005).

${ }^{10}$ X. F. Wang and P. Vasilopoulos, Phys. Rev. B 72, 165336 (2005).

${ }^{11}$ S. Bellucci and P. Onorato, J. Phys.: Condens. Matter 19, 395020 (2007); Phys. Rev. B 78, 235312 (2008).

${ }^{12}$ G. Cohen, O. Hod, and Eran Rabani, Phys. Rev. B 76, 235120 (2007).

${ }^{13}$ A. G. Aronov and Y. B. Lyanda-Geller, Phys. Rev. Lett. 70, 343 (1993).

${ }^{14}$ F. E. Meijer, A. F. Morpurgo, and T. M. Klapwijk, Phys. Rev. B 66, 033107 (2002).

${ }^{15}$ J. S. Sheng and K. Chang, Phys. Rev. B 74, 235315 (2006).

${ }^{16}$ M. Wang and K. Chang, Phys. Rev. B 77, 125330 (2008).

${ }^{17}$ M. P. Nowak and B. Szafran, Phys. Rev. B 80, 195319 (2009).

${ }^{18}$ T. Morimoto, Y. Hatsugai, and H. Aoki, Phys. Rev. B 78, 073406 (2008).

${ }^{19}$ P. Földi, O. Kalman, and M. G. Benedict, Phys. Rev. B 82, 165322 (2010).

${ }^{20}$ B. H. Wu and J. C. Cao, Phys. Rev. B 74, 115313 (2006). 
${ }^{21}$ M. P. Nowak, B. Szafran, and F. M. Peeters, Phys. Rev. B 84, 235319 (2011).

${ }^{22}$ J. Nitta, T. Akazaki, H. Takayanagi, and T. Enoki, Phys. Rev. Lett. 78, 1335 (1997)

${ }^{23}$ J. Schliemann, J. C. Egues, and D. Loss, Phys. Rev. Lett. 90, 146801 (2003).

${ }^{24}$ B. A. Bernevig, J. Orenstein, and S. C. Zhang, Phys. Rev. Lett. 97, 236601 (2006).

${ }^{25}$ J. D. Koralek, C. Weber, J. Orenstein, B. A. Bernevig, S. Zhang, S. Mack, and D. Awschalom, Nature (London) 458, 610 (2009).

${ }^{26}$ M. P. Nowak and B. Szafran, Phys. Rev. B 82, 165316 (2010).

${ }^{27}$ M. P. Nowak, B. Szafran, F. M. Peeters, B. Partoens, and W. J. Pasek, Phys. Rev. B 83, 245324 (2011).
${ }^{28}$ M. Nita, B. Ostahie, D. C. Marinescu, A. Manolescu, and V. Gudmundsson, e-print arXiv:1111.2949 (to be published).

${ }^{29}$ D. Frustaglia and K. Richter, Phys. Rev. B 69, 235310 (2004).

${ }^{30}$ M. Bayer, M. Korkusinski, P. Hawrylak, T. Gutbrod, M. Michel, and A. Forchel, Phys. Rev. Lett. 90, 186801 (2003).

${ }^{31}$ W. Knap, C. Skierbiszewski, A. Zduniak, E. Litwin-Staszewska, D. Bertho, F. Kobbi, J. L. Robert, G. E. Pikus, F. G. Pikus, S. V. Iordanskii, V. Mosser, K. Zekentes, and Yu. B. Lyanda-Geller, Phys. Rev. B 53, 3912 (1996).

${ }^{32}$ M. P. Nowak and B. Szafran, Phys. Rev. B 83, 035315 (2011). 